\title{
Access Control for hybrid Femtocell Network based on AGV Mechanism
}

\author{
Chengmei Li, Hexiang Duan, Hongbo Ba, Xiaoning Zhang, Jianjun Wu \\ Institution of Advanced Communications, EECS, \\ Peking University, Beijing, China \\ lichengmei@gmail.com, just@pku.edu.cn
}

\begin{abstract}
As most of voice calls and data traffic originates indoors, femtocells have been one of the most promising trends in LTE, which are short-range, cost-beneficial and low-power cellular home base stations that can improve indoor coverage and voice/data quality of service (QoS). One of the major challenges for femtocell network is the access control. The hybrid access control mechanism, as a tradeoff between open and closed scenario, is the most promising access mechanism from which both users and operators benefit. Femtocell user equipments (FUEs) select femtocell access points (FAPs) according to their reported channel information which FAPs confidently own, and selfish FAPs have incentive to report larger information to win greater opportunity to be selected. Considering the aforementioned truth-telling in access control issue, this paper proposes access control scheme for hybrid femtocell network based on Arrow-d'AspremontGerard-Varet (AGV) mechanism. Close form for the payment is given. Moreover, the access control scheme is nearly optimal performances with low computational complexity compared with the optimal access scheme. Furthermore, the simulation results demonstrate that the access control scheme can be apply to hybrid femtocell network.
\end{abstract}

Keywords-Femtocell, hybrid, access control, truth-telling, AGV,

\section{INTRODUCTION}

With the development of LTE, more and more smartphone users require high speed upload and download data traffic, and thus femtocell is proposed for solve this issue. Which is short-range, cost-benefcial and lowpower cellular base stations that can improve the coverage and QoS. Recently survey indicates that, femtocell is the most promising method for indoors communicaitons [1].

Femtocell has three mode: (1) Open access mode, any user can access to any femtocell without restrict.(2) Closed access mode, only the subscriber can access the femtocell. (3) Hybrid access mode, the subscriber can access all the femtocells, the non-subscriber can only access the femtocells which do not requires subscription [2]. Among the three modes. Hybrid access mode is a tradeoff between open and closed scenarios.

However, there still exist some challenges, such as resource congestion, access control, business model, auction and so on. Considering these challenges, many solution have been proposed [3]-[6]. In [3], authors studied resource sharing and femtocell access control in OFDMA femtocell networks. They designed a resource allocation mechanism that gathers selfish users' true private traffic information to achieves efficient and fair resource sharing.In [4], authors studied the business model of femtocell deployment. They modeled the interactions between a cellular operator and users as a Stackelberg game, and derived the equilibrium pricing and capacity allocation decisions.In [5], the paper combined the pricing strategy and the spectrum allocation strategy. And proposed an economic framework to improve the revenue of Wireless Serviece Provider in femtocell networks. In [6], the paper considered the lack of incentives for privately-owned femtocells to serve unregistered users is the obstacle constraining the potential capability of femtocells.

In this paper, we describe a model OFDMA femtocell network in hybrid access mode. Open FUEs can only access the open FAPs, while closed FUEs can access both open FAPs and closed FAPs in its own closed subscriber group. The model is then formulated and analyzed, and the access control scheme is described based on the AGV auction by introducing a transfer payment to realize truth-telling mechanism. We theoretically prove that in such a case the FAPs will tell their true capacities to FUEs, and there are no extra costs from the network. Besides, by using a simple method that FAPs allocate capacities to FUEs with the largest values, the complexity for such a access control scheme can be greatly decreased compared with the optimal access scheme.

The rest of the paper is organized as follows. In section II we describe the system model of hybrid access mode. In section III we present the details of our proposed access control scheme and prove the validity of AGV mechanism in hybrid access scenarios. Simulation results and conclusion are given in Sections IV and V, respectively.

\section{System Model}

In the femtocell network, the FAPs report their capacities to FUEs who have the authority to access them, and the FUEs then select the FAPs that have the largest capacities. The main base station (MBS) and all FAPs contain $K$ subchannels with bandwidth $W$ on the same frequency band. Thus there exists interference between each UE. We assume the UEs can only access the femtocells. In the hybrid access scenario, as shown in Fig1 [7], there are two kinds of FAPs, open FAPs and closed FAPs. In our model, we consider that there are $N_{O}$ open FAPs (denoted by $F_{1}, F_{2}, \cdots, F_{N_{O}}$ ) and $N_{C}$ closed ones (denoted by $F_{N_{O}+1}, F_{N_{O}+2}, \cdots, F_{N_{O}+N_{C}}$ ). The total number of FAPs is $N=N_{O}+N_{C}$. All FUEs can access open FAPs freely and they do not need to register 


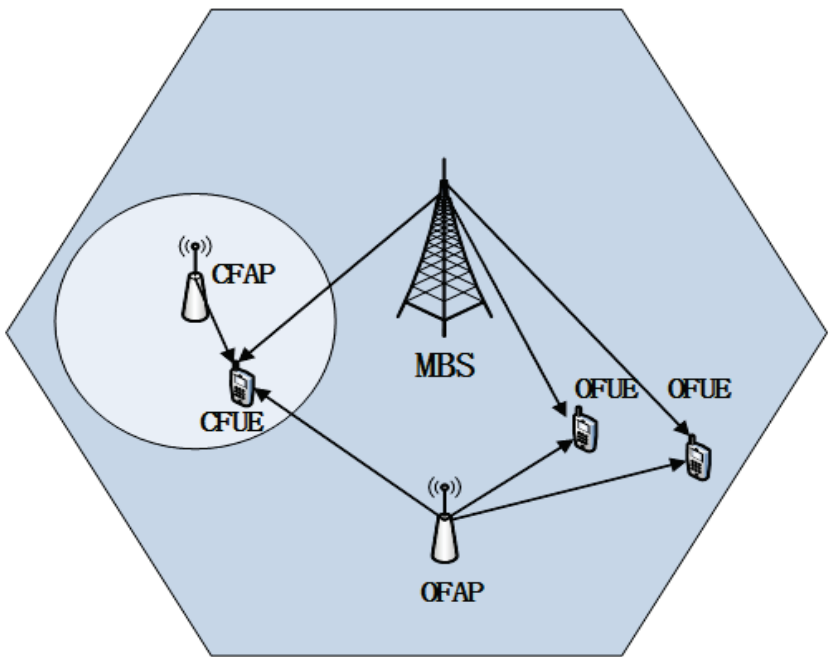

Fig. 1. Hybrid femtocell network system model.

for the accessing. On the other hand, only FUEs registered for the specific closed FAPs can access them, while those unregistered FUEs can not. Correspondingly, there are $M_{O}$ open FUEs (denoted by $U_{1}, U_{2}, \cdots, U_{M_{O}}$ ) which only have the authority to access the open FAPs, and $M_{C_{i}}$ closed FUEs (denoted by $U_{M^{n-1}+1}, U_{M^{n-1}+2}, \cdots, U_{M^{n}}$,) registered for the closed FAP $F_{N_{O}+n}$, where $n=1,2, \cdots, N_{C}$ and $M^{n}=M_{O}+\sum_{i=1}^{n} M_{C_{i}}$.

At the beginning, FAPs report their channel information to FUEs. Then, according to the reported channel information, FUEs select FAPs to connect with. Finally, FAPs allocate their subchannels to the linked FUEs based on their real subchannel information. Each FUE pays for the wireless service provided by the FAP based on the sum of the subchannel capacities they occupy. We assume that each FUE can only get access to one FAP using several subchannels to obtain information and there is no permission that two or more FUEs use the same subchannel of one FAP at the same time. Under these conditions, We aim at maximizing the throughput of the network.

The average real subchannel capacities and the reported subchannel capacities of $F_{n}$ to $U_{m}$ are defined as $\tilde{C}_{n, m}$ and $\hat{C}_{n, m}$, respectively. FUEs pay FAPs for the subchannel resource. We define $\pi$ as the unit price per bit of channel capacity. The payment of $F_{n}$ paid by $U_{m}$ can be expressed as

$$
R_{n, m}=\sum_{k \in \kappa_{n, m}} \pi \tilde{C}_{n, m, k}, \quad F_{n} \text { is selected by } U_{m},
$$

The total payment of $F_{n}$ can be expressed as

$$
R_{n}=\sum_{m=1}^{M} R_{n, m}
$$

During the access control process, the objectives of FAPs and FUEs are different. FAPs strive for financial gains, while the FUEs prefer larger channel capacities in order to obtain better communication quality. We assume that each FUE only selects one FAP according to the reported channel information. Since FAPs decide subchannel allocation, FUEs do not know which subchannels will be allocated to them. They only know that FAPs with larger average subchannel capacity have larger opportunity to provide better communication quality. Evidently, each FUE selects the FAP with the largest average value of reported subchannel capacity to guarantee itself an optimal or at least a suboptimal result.

\section{ACCESS CONTROL SCHEME FOR HYBRID SCENARIO}

In this section we present the access control scheme for the hybrid scenario described in the previous section [8]. We assume that the capacity $C_{n, m}$ that FAP $F_{n}$ reports to FUE $U_{m}$ obey the probability density function which is expressed as $f\left(\tilde{C}_{n, m}\right)$. Because $U_{m}$ selects FAPs according to their reported average subchannel capacities, $F_{n}$ does not know whether $U_{m}$ will select it or not. It can only calculate its expected payment from $U_{m}$ to be

$$
\mathcal{R}_{n, m}\left(\hat{C}_{n, m}\right)=\pi \tilde{C}_{n, m} P(n, m)
$$

where $P(n, m)$ is the probability of $F_{n}$ selected by $U_{m}$ and when $\hat{C}_{n, m} \rightarrow \infty, P(n, m) \rightarrow 1$. Therefore, $F_{n}$ has an incentive to report larger average subchannel capacity because it would lack payments if it is not selected by FUEs. In this paper, we use AGV mechanism to make the FAPs report real capacities to FUEs.

In hybrid scenario, there exist three different communication mode:(1) Open FAPs report capacities to open FUEs(2) open FAPs report capacities to closed FUEs (3) closed FAPs report capacities to closed FUEs. AGV mechanism is achieved by adding a transfer payment $\mathcal{T}_{n, m}$ to the expected revenue $\mathcal{R}_{n, m}$. The transfer payment for the three mode is different, each FAP can only get its maximum expected total payment when it reports the real information. Any cheating leads to economic losses for FAPs and the throughput of the network.

\section{A. Open FAPs report capacities to open FUEs}

the transfer payment added to $\mathcal{R}_{n, m}$ is

$$
\begin{array}{r}
\mathcal{T}_{n, m}\left(\hat{C}_{1, m}, \cdots, \hat{C}_{N_{O}, m}\right)=\Gamma_{n, m}\left(\hat{C}_{n, m}\right) \\
-\frac{1}{N_{O}-1} \sum_{i=1, i \neq n}^{N_{O}} \Gamma_{i, m}\left(\hat{C}_{i, m}\right) .
\end{array}
$$

where the externality is

$$
\Gamma_{n, m}\left(\hat{C}_{n, m}\right)=\sum_{i=1, i \neq n}^{N_{O}} E\left[\mathcal{R}_{i, m}\left(\hat{C}_{n, m}\right)\right]
$$

the expected total payment of $F_{n}$ from $U_{m}$ is

$E\left[\mathcal{U}_{n, m}\left(\hat{C}_{n, m}\right)\right]=E\left[\mathcal{R}_{n, m}\left(\hat{C}_{n, m}\right)+\mathcal{T}_{n, m}\left(\hat{C}_{1, m}, \cdots, \hat{C}_{N_{O}, m}\right)\right]$. 
inserting the Eq. (4) into the above equation, we get

$$
\begin{array}{r}
E\left[\mathcal{U}_{n, m}\left(\hat{C}_{n, m}\right)\right]=E\left[\sum_{i=1}^{N_{O}} \mathcal{R}_{i, m}\left(\hat{C}_{n, m}\right)\right] \\
-\frac{1}{N_{O}-1} \sum_{j=1, j \neq n}^{N} \Gamma_{j, m}\left(\hat{C}_{j, m}\right) .
\end{array}
$$

\section{B. open FAPs report capacities to closed FUEs}

the transfer payment added to $\mathcal{R}_{n, m}$ is

$$
\begin{gathered}
\mathcal{T}_{n, m}\left(\hat{C}_{1, m}, \cdots, \hat{C}_{N_{O}, m}, \hat{C}_{N_{O}+q, m}\right)=\Gamma_{n, m}\left(\hat{C}_{n, m}\right) \\
-\frac{1}{N_{O}}\left[\sum_{i=1, i \neq n}^{N_{O}} \Gamma_{i, m}\left(\hat{C}_{i, m}\right)+\Gamma_{N_{O}+q, m}\left(\hat{C}_{N_{O}+q, m}\right)\right] .
\end{gathered}
$$

where the externality is

$$
\begin{aligned}
\Gamma_{n, m}\left(\hat{C}_{n, m}\right)= & \sum_{i=1, i \neq n}^{N_{O}} E\left[\mathcal{R}_{i, m}\left(\hat{C}_{n, m}\right)\right] \\
& +E\left[\mathcal{R}_{N_{O}+q, m}\left(\hat{C}_{n, m}\right)\right] . \\
\Gamma_{N_{O}+q, m}\left(\hat{C}_{N_{O}+q, m}\right)= & \sum_{i=1}^{N_{O}} E\left[\mathcal{R}_{i, m}\left(\hat{C}_{N_{O}+q, m}\right)\right] .
\end{aligned}
$$

the expected total payment of $F_{n}$ from $U_{m}$ is

$$
\begin{aligned}
& E\left[\mathcal{U}_{n, m}\left(\hat{C}_{n, m}\right)\right]=E\left[\sum_{i=1}^{N_{O}} \mathcal{R}_{i, m}\left(\hat{C}_{n, m}\right)+\mathcal{R}_{N_{O}+q, m}\left(\hat{C}_{n, m}\right)\right] \\
& -\frac{1}{N_{O}}\left[\sum_{j=1, j \neq n}^{N} \Gamma_{j, m}\left(\hat{C}_{j, m}\right)+\Gamma_{N_{O}+q, m}\left(\hat{C}_{N_{O}+q, m}\right)\right] .
\end{aligned}
$$

\section{C. closed FAPs report capacities to closed FUEs}

the transfer payment added to $\mathcal{R}_{n, m}$ is

$$
\begin{aligned}
& \mathcal{T}_{N_{O}+q, m}\left(\hat{C}_{1, m}, \cdots, \hat{C}_{N_{O}, m}, \hat{C}_{N_{O}+q, m}\right) \\
& =\Gamma_{N_{O}+q, m}\left(\hat{C}_{N_{O}+q, m}\right)-\frac{1}{N_{O}} \sum_{i=1}^{N_{O}} \Gamma_{i, m}\left(\hat{C}_{i, m}\right) .
\end{aligned}
$$

where the externality is the same as OC case.

the expected total payment of $F_{n}$ from $U_{m}$ is

$$
\begin{aligned}
& E\left[\mathcal{U}_{N_{O}+q, m}\left(\hat{C}_{N_{O}+q, m}\right)\right] \\
& =E\left[\sum_{i=1}^{N_{O}} \mathcal{R}_{i, m}\left(\hat{C}_{N_{O}+q, m}\right)+\mathcal{R}_{N_{O}+q, m}\left(\hat{C}_{N_{O}+q, m}\right)\right] \\
& -\frac{1}{N_{O}}\left[\sum_{j=1, j \neq n}^{N} \Gamma_{j, m}\left(\hat{C}_{j, m}\right)\right] .
\end{aligned}
$$

The first terms in the right side of Eqs. (7), (11), (13) represent the expected payment of all FAPs paid by $U_{m}$ when $F_{n}$ reports $\hat{C}_{n, m}$ to $U_{m}$. Since other terms are independent of $\hat{C}_{n, m}$ to $U_{m}$, only the first term determines the expected total payment of $F_{n}$ paid by $U_{m}$. According to Eq. (3), the expected payment of $F_{n}$ from $U_{m}$ is determined by the reported information which may not be true. Only when all FUEs select FAPs based on the true information can the whole network get the largest expected total payment from FUEs. Any cheating leads to a decrease in the expected total payment. Therefore, $E\left[\mathcal{U}_{n, m}\left(\hat{C}_{n, m}\right)\right]$ can reach its maximum value when $F_{n}$ reports its true information $\left(\hat{C}_{n, m}=\tilde{C}_{n, m}\right)$. Thus, $F_{n}$ has no incentive to report false information and the equilibrium is achieved.

\section{Simulation Results}

To validate the performances of proposed access control scheme performances, we conduct the following simulations. We assume that the reported subchannel capacity of each FAP obeys the density function: $f(x)=e^{-x}$. We follow the principle that the FUEs choose the FAP with the largest reported average channel capacities. Each FAPs only know his own payment from each FUEs.

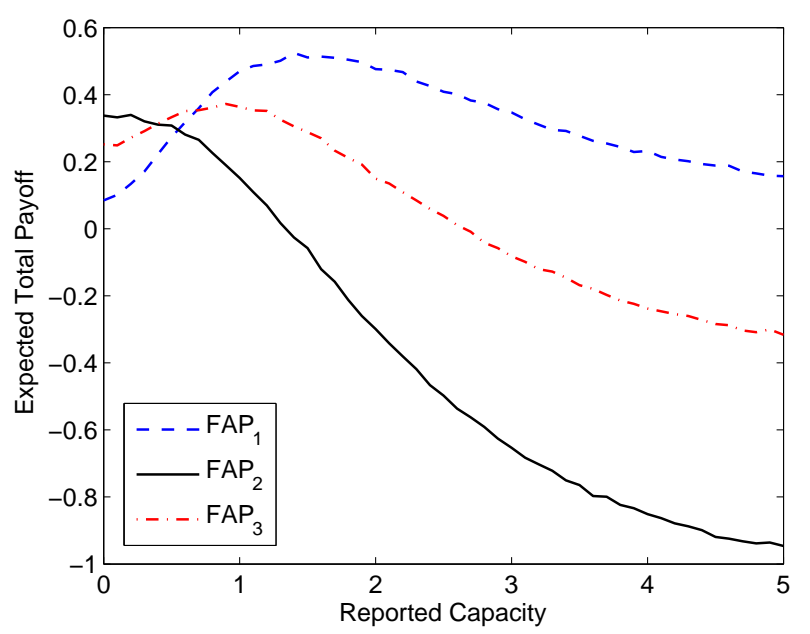

Fig. 2. Expected total payment when different average subchannel capacities are reported to $U_{4}$.

In the hybrid scenario, we consider the open FAPs $N_{O}=2$, open FUEs $M_{O}=3$, closed FAPs $N_{C}=1$, closed FUEs $M_{C}=M_{C_{1}}=1, M_{O}=5$, open FUEs $N_{O}=2$, We also assume that the price per unit of channel capacity $\pi=1$ and a random sample of the real average channel capacity is obtained as

$$
C=\left(\begin{array}{ccc}
\tilde{C}_{1,1} & \tilde{C}_{2,1} & - \\
\tilde{C}_{1,2} & \tilde{C}_{2,2} & - \\
\tilde{C}_{1,3} & \tilde{C}_{2,3} & - \\
\tilde{C}_{1,4} & \tilde{C}_{2,4} & \tilde{C}_{3,4}
\end{array}\right)=\left(\begin{array}{ccc}
0.87 & 1.2 & - \\
0.33 & 1.92 & - \\
9.08 & 2.38 & - \\
1.51 & 0.14 & 0.87
\end{array}\right) .
$$


$U_{1}, U_{2}, U_{3}$ are open FUEs, $U_{4}$ is closed FUE, without loss of generality, we consider $U_{4}$ as example. Fig.2 demonstrate the variation of the expected total payment of each FAP by $U_{4}$. The expected payment varies with the reported capacity. And there exists a peak. That because FAP can only get the maximum expected payment when it report the truth capacity. In Fig.2, the open FAP1, FAP2, closed FAP3 get their maximum expected total payments $0.5242,0.3394,0.3723$, respectively, at their real capacities $\tilde{C}_{1,4}=1.51, \tilde{C}_{2,4}=0.14$ and $C_{3,4}=0.87$ for FUE $U_{4}$. FUE will choose the FAP which has the largest reported capacities as it has more probability to provider larger capacity. So $U_{4}$ will choose FAP1, thus FAP1 can get the largest expected total payment 0.5242.The expected total payment is less than $\pi \tilde{C}_{1,4}=1.51$ because it needs to pay transfer payment to other FAPs.

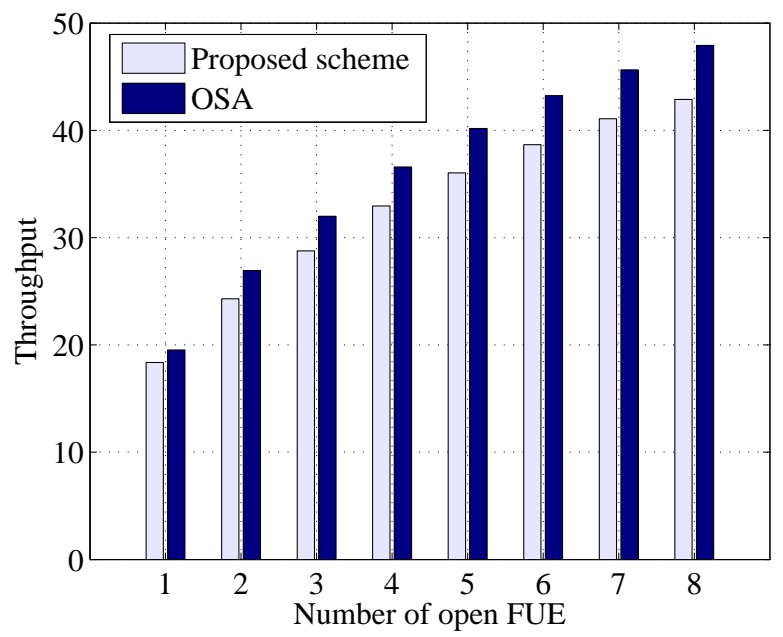

Fig. 3. Throughput comparison at different $M_{O}$.

Fig. 3 compare our proposed scheme with the OSA scheme. we set $N_{O}=2, N_{C}=1, M_{C}=1$, then change the value of $M_{O}$ to investigate the performance in different scenario. As the number of open FUEs increases, the FAPs are used more efficiently, the whole throughput of the network becomes larger. And from Fig.3, we can see our proposed scheme can get nearly the performance as OSA scheme even in different number of open FUEs. The running time of the proposed scheme is $O(N M K)$ and the OSA shcme is $O\left(N^{M} K\right)$. Thus demonstrates our proposed scheme has the lower computational complexity while the simulation results near the OSA scheme.

\section{Conclusion}

In this paper, we have have investigated the access control scheme, which mainly solves the access and truth-telling issues. The proposed scheme is realized by adding a transfer payment to balance the total payment. And the FUE choose the FAP who report the largest capcity. Thus the proposed scheme can make sure that FAPs has no incentive to report fake information. Then we compare the performance of proposed scheme with the OSA scheme. Finally, The simulation results demonstrate our proposed scheme can get the near optimal performance while has the lower computational complexity .

\section{ACKNOWLEDGMENT}

This work is partly supported by the National Science Foundation of China (Grant No. NFSC \#61071083, \#61371073) and the National High-Tech Research and Development Program of China (863 Program), No.2012AA01A506. Corresponding author: Jianjun Wu, E-mail: just@pku.edu.cn.

\section{REFERENCES}

[1] H. Claussen, L. T. W. Ho, and L. G. Samuel, "An overview of the femtocell concept," Bell Labs Tech.J., vol. 13, no. 1, pp. 221-246, May. 2008.

[2] Assen Golaup, Mona Mustapha, Leo Boonchin Patanapongpibul and Vodafone Group, "Femtocell access control strategy in UMTS and LTE," IEEE Communications Magazine, vol. 47, no. 9, pp. 117-123, Sep. 2009.

[3] C. Ko and H. Wei, "On-Demand Resource-Sharing Mechanism Design in Two-Tier OFDMA Femtocell Networks," IEEE Transactions on Vehicular Technology, vol. 60, no. 3, pp. 1059-1071, Mar 2011.

[4] L. Duan, J. Huang, and B. Shou, "Economics of Femtocell Service Provision," IEEE Transactions on Mobile Computing,Sep 2012.

[5] Y. Chen,J. Zhang,P. Lin and Q. Zhang, "Optimal Pricing and Spectrum Allocation for Wireless Service Provider on Femtocell Deployment," in Proc. IEEE ICC, Jun. 2011.

[6] S. Hua, X. Zhuo, and Shivendra. S. Panwar, "A Truthful Auction based Incentive Framework for Femtocell Access," in Proc. IEEE WCNC, Jan. 2013.

[7] Poongup Lee, Taeyoung Lee, Jangkeun Jeong, and Jitae Shin, "Interference management in LTE femtocell systems using Fractional Frequency Reuse," in Proc. IEEE ICACT, Feb. 2010.

[8] Ziqiang Feng, Lingyang Song, Zhu Han, Dusit Niyato, and Xiaowu Zhao, "Cell Selection in Two-Tier Femtocell Networks with Open/Closed Access Using Evolutionary Game," in Proc. IEEE WCNC, Apr. 2013.

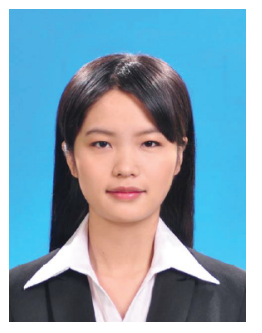

Chengmei Li received her bachelor degree in electrical engineering from Beijing University of Aeronautics and Astronautics, Beijing, P.R. China, in 2011. Since 2011, she has been a postgraduate student in Institution of Advanced Communications, Peking University, China. Her research interests are in the area of satellite mobile communications compatible with LTE. Email: lichengmei1989@gmail.com.

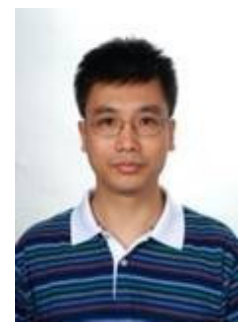

Jianjun Wu received his B.S., M.S. and Ph.D. degree from Peking University, Beijing, P. R. China, in 1989, 1992 and 2006, respectively. Since 1992, he has joined the School of Electronics Engineering and Computer Science, Peking University, and has been appointed as an associate professor since 2002. His research interests are in the areas of satellite communications, wireless communications, and communications signal processing. *The corresponding author. Email: just@pku.edu.cn. 